\title{
La producción científica en tesis doctorales sobre aprendizaje servicio en España (2000-2016)
}

\author{
Elena Fernández Rey, Rebeca García Murias \\ Universidad de Santiago de Compostela, España
}

\section{Resumen}

El interés por conocer las tendencias de las investigaciones realizadas en el ámbito universitario sobre aprendizaje servicio (ApS) en las dos últimas décadas en el contexto español ha llevado a indagar las Tesis Doctorales sobre esta temática en el período comprendido entre 2000 y 2016. A partir de los datos recogidos en la Base de datos TESEO del Ministerio de Educación, Cultura y Deporte se ha realizado un análisis de contenido de los títulos, descriptores y resúmenes, valorando la productividad del área, las principales características generales y metodológicas y las temáticas estudiadas. El estudio ha mostrado el interés creciente en el ámbito académico y científico por el aprendizaje servicio, ya que se trata de una metodología pedagógica que promueve el desarrollo de diversas competencias a través de actividades educativas que incluyen el servicio a la comunidad. El contexto universitario destaca como uno de los prioritarios para el desarrollo de programas de ApS.

\section{Palabras clave}

Aprendizaje servicio, educación universitaria, tesis doctoral.

Fecha de recepción: 9/II/2017

Fecha de aceptación: 9/III/2017 


\title{
Scientific production in Doctoral Thesis on Service Learning in Spain (2000-2016)
}

\author{
Abstract \\ The interest in knowing the trends of research conducted at the university level on \\ service learning in the last two decades in the Spanish context has led to inquire the \\ Doctoral Thesis on this topic in the period between 2000 and 2016. From the data \\ collected into the TESEO database from the Ministry of Education, Culture and Sport it \\ has been done a content analysis of the titles, descriptors and abstracts, assessing the \\ productivity of the area, the main general and methodological characteristics and the \\ thematic studied. The study has shown increasing interest in the academic and \\ scientific field onto service learning as it is a teaching methodology that promotes the \\ development of various competencies through educational activities including \\ community service. The university context stands as one of the priority for the \\ development of SL programs.
}

\section{Keywords}

Service learning, Higher Education, Doctoral Thesis. 


\section{Introducción}

El modelo de aprendizaje servicio encierra un gran potencial pedagógico, posibilitando la adquisición de competencias básicas a partir de los proyectos de trabajo que se lleven a cabo.

El aprendizaje servicio es un método de enseñar y de aprender. El proceso de enseñanza-aprendizaje se desarrolla a través de la realización de un servicio a la comunidad, por lo que se trata de un instrumento pedagógico y de una herramienta que puede ayudar a mejorar la educación.

En este sentido el ApS contribuye al desarrollo competencial del alumnado de cualquier etapa educativa del siguiente modo:

- Aportando un conocimiento más profundo de los retos y problemáticas sociales, de sus causas y consecuencias.

- Posibilitando una visión más amplia de la realidad.

- Facilitando un mejor conocimiento del tejido asociativo solidario y de las personas comprometidas con el entorno para su transformación social.

- Desarrollando habilidades relacionadas con la realización de proyectos.

- Descubriendo destrezas y aptitudes individuales para ponerlas al servicio de la comunidad.

\section{Rilis \\ Revista Iberoamericana de Aprendizaje Servicio}

- Desarrollando valores que favorecen la autonomía personal y que mejoran la coherencia personal: solidaridad, responsabilidad, justicia, igualdad, etc.

- Mejorando las capacidades para trabajar en equipo.

- Desarrollando actitudes sociales y hábitos de convivencia.

El interés particular en este trabajo de investigación se centró en conocer el impacto que posee el uso de esta metodología fundamentada en el aprendizaje experiencial cooperativo, crítico y asociado a valores integrando el servicio comunitario solidario con el aprendizaje de competencias curriculares, de modo que los estudiantes aprendan y se desarrollen integralmente como personas, profesionales y ciudadanos mediante la realización de un servicio útil para la comunidad y la reflexión crítica de la experiencia, especialmente con el alumnado universitario.

Consideramos que la relevancia de esta metodología se verá reflejada en la disposición en el ámbito de la investigación por abordar esta temática. Por ello, los objetivos de este estudio son:

- Analizar la producción científica en Tesis Doctorales sobre aprendizaje servicio en España entre 2000-2016.

- Conocer las tendencias de las investigaciones realizadas en el ámbito universitario sobre el aprendizaje servicio en las últimas 
décadas en el contexto español.

\section{Marco teórico}

En la actual sociedad de la información que nos caracteriza, nuestro sistema educativo requiere de nuevas formas de enseñanza-aprendizaje que respondan a las demandas actuales. No se trata tan sólo de aprender a conocer, sino también de aprender a hacer, aprender a vivir y aprender a ser (Delors, 1996).

En este contexto, las universidades europeas han culminando la configuración de las titulaciones de grado y de máster con un nuevo modelo conceptualizado, el aprendizaje basado en competencias o aprendizaje por competencias definido como la combinación de habilidades prácticas, conocimientos, motivación, valores éticos, actitudes, emociones y otros componentes sociales que se movilizan de forma conjunta con la finalidad de lograr una acción eficaz (Hersh, Simone, Moser y Konstant, 1999).

Se parte de una visión y misión de la universidad como espacio y actor transformador de la sociedad, con la presencia general de elementos educativos claves que impulsen una formación para la ciudadanía global y no que proporcione tan solo una oferta limitada a un ámbito formativo especializado o para un grupo de profesionales específicos. El proceso de enseñanza-aprendizaje progresivamente se vuelve más complejo y requiere de nuevas metodologías y propuestas educativas. Una buena herramienta educativa para dar respuesta a estas demandas es el denominado aprendizaje servicio (ApS) ya que, a través de ella, se aglutina el aprendizaje de diferentes competencias básicas o específicas, como son el trabajo en equipo interdisciplinar, las habilidades en las relaciones personales, el compromiso ético o el razonamiento crítico (ANECA, 2005).

Teniendo en cuenta este escenario las universidades españolas han realizado un importante esfuerzo para la adaptación al Espacio Europeo de Educación Superior (EEES), cuya concreción y desarrollo se ha vinculado al discurso de las competencias y la empleabilidad. Este nuevo enfoque ha implicado una renovación curricular de las titulaciones universitarias, requiriendo un cambio metodológico en la formación universitaria, y como consecuencia, en la evaluación (Bolívar, 2008).

El desarrollo de un enfoque metodológico dirigido a la adquisición de competencias supone funcionalidad y relevancia de los contenidos, aplicación de lo que se aprende. Por tanto, las actividades educativas a desarrollar por el alumnado han de estar dirigidas a conseguir las competencias deseables y los resultados de aprendizaje previstos, y para ello, deben estar relacionadas con la vida cotidiana y profesional.

En este contexto la incorporación de metodologías educativas innovadoras como el ApS, entendida como "una forma de educación basada en la experiencia donde los estudiantes se comprometen en actividades que relacionan las necesidades personales y 
de la comunidad con oportunidades intencionalmente diseñadas para promover el desarrollo y el aprendizaje del alumnado" (Jacoby, 1996, 5), han demostrado altos niveles de efectividad y eficiencia, identificando, entre sus posibles beneficios, el aumento del compromiso del alumnado, la mejora del aprendizaje académico, el fomento del desarrollo personal y social de los estudiantes, el incremento de la motivación del profesorado y los cambios de sus actitudes hacia la enseñanza, etc., los cuales aportan evidencias con las que fundamentar el modelo de aprendizaje basado en competencias y contribuyen al cumplimiento de las exigencias que plantea el proceso de convergencia europeo de educación superior.

El ApS es una propuesta educativa que combina procesos de aprendizaje y de servicio a la comunidad a través de un único proyecto bien articulado donde el alumnado participante aprende simultáneamente que trabaja en necesidades reales del entorno con el propósito de mejorarlo (Puig, Batlle, Bosch y Palos, 2007).

El ApS entiende la educación como la formación de ciudadanos activos que aportan sus conocimientos y valores al desarrollo y al progreso del medio social donde se desenvuelven. Este modelo de aprendizaje se articula en torno a dos ideas básicas: por una parte, el aprendizaje mejora el servicio de modo que lo que se aprende permite prestar un servicio de calidad a la comunidad y por otro, el servicio motiva y dota de sentido al aprendizaje, aportándole una experiencia que posibilita extraer nuevos aprendizajes.

Se fundamenta en una metodología reflexiva, relacional y estructurada que combina una metodología al servicio de la comunidad con numerosas oportunidades para el aprendizaje. Se basa en la educación experimental como base para el crecimiento intelectual, moral y cívico.

Entre sus principales requisitos destaca el entender la intencionalidad educativa del aprendizaje como una posibilidad de realizar un servicio a la comunidad, a través de la participación activa y la reflexión.

Tal y como apuntan Piqué y Forés (2012) entre sus principales potencialidades destacan la apertura y proyección de las instituciones educativas al entorno social; detecta y actúa sobre las necesidades reales de la comunidad; supone un servicio a la comunidad para aprender y colaborar en un marco de reciprocidad; pone en juego contenidos curriculares y competencias para la vida; se inspira en una pedagogía de la experiencia, la participación activa, la interdisciplinariedad, el trabajo en equipo y la reflexión; se basa en la coordinación entre instituciones educativas, organizaciones sociales e instancias del entorno y posee un impacto educativo y transformador múltiple.

Un aspecto relevante del aprendizaje servicio es que sus aprendizajes posibilitan el desarrollo de numerosas competencias, integrando así capacidades, habilidades, conocimientos 
y valores que permiten resolver situaciones reales eficazmente.

Un buen desarrollo de estas competencias activa una ciudadanía responsable, sobre todo cuando el aprendizaje y desarrollo de estas competencias es realizado por medio de acciones de interés social.

Siguiendo a Puig, Batlle, Bosch y Palos (2007) algunos de los aspectos metodológicos fundamentales para el desarrollo competencial, reflejados en el ApS, son los que siguen:

1. Se desarrolla en contextos concretos: contextualización de los aprendizajes -situaciones reales que dotan de sentido y funcionalidad a los aprendizajes-.

2. Aborda el aprendizaje de la complejidad: enfrenta al alumnado a situaciones tan complejas como su nivel de desarrollo aconseje, dotándole de estrategias para abordarlas con eficacia.

3. Promueve el protagonismo del alumnado: desarrolla su iniciativa personal, potencia la toma de decisiones y actitudes emprendedoras.

4. Estimula el trabajo cooperativo: la actividad en grupos cooperativos permite compartir el trabajo entre iguales, estimulando el aprendizaje activo, constructivo y autónomo; facilita la comunicación interpersonal y proporciona al alumnado confianza en sí mismo y en el grupo y favorece un mejor rendimiento escolar.
5. Requiere una revisión crítica de los currículos: identificar y priorizar los temas socialmente relevantes como elementos vertebradores de los proyectos de ApS.

6. Precisa nuevas formas de organización escolar: flexibilización de las estructuras escolares (horarios, espacios, agrupamientos, etc.). Permite el trabajo dentro y fuera del aula.

7. No se reduce a un mero proyecto de carácter procedimental y de adquisición de habilidades sino que se integran componentes educativos del aprender a ser y aprender a convivir, que procuran el bienestar emocional individual y colectivo. El alumnado aprende a desarrollarse como persona.

2.1. El aprendizaje servicio en España: aspectos legislativos

En España se comienza a hablar de aprendizaje servicio desde hace relativamente poco tiempo. A pesar de ello, las prácticas en los centros educativos y entidades sociales poseen una larga tradición en nuestro país.

Algunos espacios donde se desarrollan estas iniciativas tienen lugar en los centros de educación primaria, secundaria, educación especial o formación profesional, universidades, entidades sociales, movimientos juveniles y de educación del tiempo libre, e instituciones de inserción social de personas adultas en riesgo de exclusión. 
En ocasiones, estas prácticas no son reconocidas como prácticas de aprendizaje servicio en sí mismas sino que se suman a los objetivos de aprendizaje y de servicio a la comunidad. Otras veces, se llevan a cabo sin ninguna vinculación curricular o sin un objetivo formativo preciso, sino más bien como experiencias de voluntariado. Es decir, no son propiamente aprendizaje servicio ya que el componente del aprendizaje intencional no está presente, no obstante, pueden considerarse un buen precedente.

En el contexto español se procuró que el sector educativo y social no interpretase el ApS como un invento sofisticado ajeno a su cultura sino como un recurso innovador que permitiese revalorizar las buenas prácticas que se habían venido desarrollando hasta entonces aunque con otra denominación, y que se posibilitase el establecimiento de una vinculación entre los aprendizajes curriculares y el servicio a la comunidad.

Para ello se trató de situar el ApS en el centro de las organizaciones ya existentes en cada territorio o comunidad autónoma, estimulando que fuese identificado como útil para sus fines y se encontrase sentido a la hora de aunar esfuerzos y coordinarse con otros agentes del entorno para promoverlo más allá de su propio ámbito de actuación.

A continuación, se presentan algunas referencias legislativas de ámbito estatal que abordan el aprendizaje servicio en los ámbitos universitario y del voluntariado, por la relevancia que en éstos puede alcanzar esta metodología.

En el sector de la educación superior, el Real Decreto 1791/2010, de 30 de diciembre, del Estatuto del Estudiante Universitario incluye en el artículo 64 de su capítulo XIV, referido a las actividades de participación social y cooperación al desarrollo de los estudiantes, una serie de principios generales. Entre los que sobresalen los siguientes:

La labor de la universidad en el campo de la participación social y la cooperación al desarrollo está vinculada a su ámbito propio de actuación (docencia, investigación y transferencia de conocimiento), cuestiones esenciales tanto para la formación integral de los estudiantes, como para una mejor comprensión de los problemas actuales.

- Como derechos de los estudiantes se especifican la incorporación de actividades de participación social y cooperación al desarrollo planificadas por la universidad; la formación gratuita para el desarrollo de estas actividades; la acreditación como voluntario y/o cooperante y la certificación de los servicios prestados.

- Como deber del alumnado se expone la participación en las acciones formativas diseñadas para un adecuado desarrollo de las propuestas de participación social y cooperación al desarrollo. 


\section{- La posibilidad de realizar el prácticum en proyectos de cooperación al desarrollo y participación social, además de favorecer prácticas de responsabilidad social y ciudadana que combinen los aprendizajes académicos de la titulación del estudiante con la prestación de servicio en la comunidad, que ayude a la mejora de la calidad de vida y la inclusión social.}

En el sector del voluntariado la Ley 45/2015, de 14 de octubre, de Voluntariado, presenta en su artículo 6 los ámbitos de actuación del voluntariado, que se centran en diez dimensiones (tabla 1). Específicamente en una de ellas, el voluntariado educativo, se propone como una de las posibilidades de actuación los programas de aprendizaje servicio; pero en muchos otros ámbitos (social, cultural, de ocio y tiempo libre, por ejemplo) también se podrían emplear este tipo de programas.

\begin{tabular}{|l|l|l|}
\hline \multicolumn{1}{|c|}{ Ámbito } & \multicolumn{1}{|c|}{ Objetivos } & \multicolumn{1}{c|}{ Acciones } \\
\hline Social & $\begin{array}{l}\text { Alcanzar una } \\
\text { mejor calidad de } \\
\text { vida y una mayor } \\
\text { cohesión y justicia } \\
\text { social }\end{array}$ & $\begin{array}{l}\text { Intervención con } \\
\text { las personas y la } \\
\text { realidad social, } \\
\text { frente a } \\
\text { situaciones de } \\
\text { vulneración, } \\
\text { privación o falta } \\
\text { de derechos u } \\
\text { oportunidades }\end{array}$ \\
\hline $\begin{array}{l}\text { Internacional } \\
\text { de } \\
\text { cooperación } \\
\text { para el } \\
\text { desarrollo }\end{array}$ & $\begin{array}{l}\text { Promocionar el } \\
\text { desarrollo en lo } \\
\text { relativo a la } \\
\text { acción } \\
\text { humanitaria y la } \\
\text { solidaridad } \\
\text { internacional }\end{array}$ & $\begin{array}{l}\text { Educación para el } \\
\text { desarrollo como } \\
\text { parte del proceso } \\
\text { educativo y de } \\
\text { transformación }\end{array}$ \\
\hline Ambiental & $\begin{array}{l}\text { Disminuir el } \\
\text { impacto negativo } \\
\text { del ser humano } \\
\text { sobre el medio } \\
\text { ambiente }\end{array}$ & $\begin{array}{l}\text { Protección y } \\
\text { recuperación } \\
\text { Conservación y } \\
\text { mejora } \\
\text { Educación y }\end{array}$ \\
\hline
\end{tabular}

\begin{tabular}{|c|c|c|}
\hline & $\begin{array}{l}\text { Poner en valor el } \\
\text { patrimonio natural } \\
\text { existente }\end{array}$ & $\begin{array}{l}\text { sensibilización } \\
\text { medioambiental }\end{array}$ \\
\hline Cultural & $\begin{array}{l}\text { Promover y } \\
\text { defender el } \\
\text { derecho de acceso } \\
\text { a la cultura y, en } \\
\text { particular, la } \\
\text { integración } \\
\text { cultural de todas } \\
\text { las personas }\end{array}$ & $\begin{array}{l}\text { Promoción y } \\
\text { protección de la } \\
\text { identidad cultural } \\
\text { Defensa y } \\
\text { salvaguarda del } \\
\text { patrimonio } \\
\text { cultural } \\
\text { Participación en } \\
\text { la vida cultural de } \\
\text { la comunidad } \\
\end{array}$ \\
\hline Deportivo & $\begin{array}{l}\text { Contribuir a la } \\
\text { cohesión } \\
\text { ciudadana y } \\
\text { social, sumando a } \\
\text { los valores } \\
\text { propios del } \\
\text { voluntariado los } \\
\text { inherentes al } \\
\text { deporte } \\
\text { Fomentar la } \\
\text { dimensión } \\
\text { comunitaria en el } \\
\text { desarrollo de la } \\
\text { práctica deportiva } \\
\text { Favorecer el } \\
\text { compromiso de } \\
\text { quienes practican } \\
\text { deporte en la vida } \\
\text { asociativa }\end{array}$ & $\begin{array}{l}\text { Paralimpismo } \\
\text { Promoción de la } \\
\text { educación e } \\
\text { inclusión social }\end{array}$ \\
\hline Educativo & $\begin{array}{l}\text { Mejorar las } \\
\text { posibilidades de } \\
\text { realización de } \\
\text { actividades } \\
\text { extraescolares y } \\
\text { complementarias } \\
\text { Contribuir a } \\
\text { compensar las } \\
\text { desigualdades } \\
\text { entre alumnos por } \\
\text { diferencias } \\
\text { sociales, } \\
\text { personales o } \\
\text { económicas }\end{array}$ & $\begin{array}{l}\text { Acción solidaria } \\
\text { planificada e } \\
\text { integrada en el } \\
\text { sistema y la } \\
\text { comunidad } \\
\text { educativa } \\
\text { Programas de } \\
\text { aprendizaje } \\
\text { servicio }\end{array}$ \\
\hline $\begin{array}{l}\text { Socio- } \\
\text { sanitario }\end{array}$ & $\begin{array}{l}\text { Ofrecer apoyo y } \\
\text { orientación a las } \\
\text { familias y al } \\
\text { entorno más } \\
\text { cercano, } \\
\text { mejorando las } \\
\text { condiciones de } \\
\text { vida }\end{array}$ & $\begin{array}{l}\text { Promoción de la } \\
\text { salud. Prevención } \\
\text { de la } \\
\text { enfermedad. } \\
\text { Asistencia } \\
\text { sanitaria. } \\
\text { Rehabilitación y } \\
\text { atención social } \\
\text { Intervención } \\
\text { integral y } \\
\text { especializada en } \\
\text { los aspectos } \\
\text { físico, psicológico } \\
\text { y social }\end{array}$ \\
\hline $\begin{array}{l}\text { De ocio y } \\
\text { tiempo libre }\end{array}$ & $\begin{array}{l}\text { Formar y } \\
\text { sensibilizar en los }\end{array}$ & $\begin{array}{l}\text { Actividades en el } \\
\text { ámbito de la }\end{array}$ \\
\hline
\end{tabular}




\begin{tabular}{|c|c|c|}
\hline & $\begin{array}{l}\text { principios y } \\
\text { valores de la } \\
\text { acción voluntaria }\end{array}$ & $\begin{array}{l}\text { educación no } \\
\text { formal que } \\
\text { fomenten el } \\
\text { desarrollo, } \\
\text { crecimiento } \\
\text { personal y grupal } \\
\text { de forma integral } \\
\text { (solidaridad, } \\
\text { inclusión, } \\
\text { compromiso, } \\
\text { participación, } \\
\text { implicación } \\
\text { social) }\end{array}$ \\
\hline Comunitario & $\begin{array}{l}\text { Favorecer la } \\
\text { mejora de la } \\
\text { comunidad } \\
\text { Promover la } \\
\text { participación con } \\
\text { mayor poder de } \\
\text { decisión e } \\
\text { iniciativa para } \\
\text { resolver los } \\
\text { problemas y } \\
\text { exigir mayor } \\
\text { calidad de vida }\end{array}$ & $\begin{array}{l}\text { Acciones en los } \\
\text { espacios vitales } \\
\text { más cercanos } \\
\text { que ayuden a } \\
\text { crear una } \\
\text { sociedad } \\
\text { solidaria, activa, } \\
\text { crítica, } \\
\text { comprometida y } \\
\text { corresponsable }\end{array}$ \\
\hline $\begin{array}{l}\text { De } \\
\text { protección } \\
\text { civil }\end{array}$ & $\begin{array}{l}\text { Colaborar } \\
\text { regularmente en } \\
\text { la gestión de las } \\
\text { emergencias, en } \\
\text { las actuaciones } \\
\text { que se } \\
\text { determinen por el } \\
\text { Sistema Nacional } \\
\text { de Protección Civil }\end{array}$ & $\begin{array}{l}\text { Participación } \\
\text { ciudadana en la } \\
\text { respuesta social a } \\
\text { estos fenómenos }\end{array}$ \\
\hline
\end{tabular}

Tabla 1: Ámbitos de actuación del voluntariado. Fuente: elaborado a partir de Ley 45/2015, de Voluntariado

\subsection{El aprendizaje servicio en el contexto universitario}

El aprendizaje servicio, particularmente en el contexto universitario, es percibido como una metodología práctica aplicable a una titulación y/o materia que aporta diferentes beneficios, dependiendo de la entidad o grupo donde se desarrolla. A través de prácticas de ApS, desde distintas titulaciones universitarias y materias curriculares, se pretende aportar un mayor significado y aplicación a los aprendizajes de los universitarios, permitiendo desarrollar contenidos curriculares, facilitando la práctica de la planificación, el esfuerzo, el compromiso, etc.

Según evidencian diversas experiencias e investigaciones sobre el tema, el ApS activa el ejercicio de todas las competencias del currículum, contemplando las competencias de iniciativa personal y competencia social y ciudadana, es decir, la responsabilidad social.

Esta metodología presenta similitudes con el trabajo de campo, pero incorporando el objetivo de hacer un servicio a la comunidad próxima, requiriendo para ello un trabajo en red con asociaciones e instituciones de la comunidad, lo cual contribuye también al compromiso social que tiene la Universidad.

La metodología de prácticas mediante aprendizaje servicio posee una fundamentación pedagógica que coincide con las demandas en el Espacio Europeo de Educación Superior que hacen referencia al constructivismo, pedagogía activa, aprendizaje experiencial, aprendizaje cooperativo, metodología globalizada y principio de la actividad asociada con la proyección social. Se trata de una actividad posible, adecuada y deseable en Educación Superior, una metodología innovadora que encaja en las propuestas de cambio actuales marcadas por el EEES.

Es un buen momento para establecer cambios y acuerdos en la manera de entender la universidad ya que se 
apuesta por un proceso de enseñanzaaprendizaje más participativo y menos individualista. Así pues, a través de experiencias como la del ApS se ofrece la posibilidad de establecer nuevos espacios de implicación en la comunidad más allá del voluntariado, experiencias donde los contenidos curriculares puedan devenir conocimientos contextualizados en la realidad, lo que hará que el servicio prestado sea de mayor calidad y los aprendizajes sean más significativos.

Las experiencias de ApS son óptimas a la hora de desarrollar las competencias transversales, tan valoradas y demandadas hoy en día en el mercado laboral (entre otras, el trabajo en equipo, la toma de decisiones, la convivencia, la autonomía, la iniciativa, las habilidades lingüísticas o las competencias tecnológicas).

Los proyectos de ApS contribuyen a impulsar una universidad de calidad, una participación ciudadana y una creación de capital social ya que éstos poseen entre sus objetivos, la mejora de algún aspecto de la sociedad, que se lleva a cabo a través de un servicio de calidad, con la participación del alumnado y de la ciudadana hacia la búsqueda del bien común, superando al individualismo e interés privados.

\section{Así, estas propuestas educativas} persiguen la participación del alumnado en la vida pública $y$, para que tengan éxito, necesitan organizar la convivencia y ofrecer la posibilidad de contribuir de manera personal en la mejora social (Puig, Batlle, Bosch et al., 2006). De este modo, el alumnado participa y se implica en un proyecto complejo que surge y se desarrolla en la realidad, de modo que se manifiestan aprendizajes que sería complicado que se diesen de otro modo, como son la sensibilidad frente a las problemáticas sociales, el compromiso que se adquiere frente al proyecto y los beneficiarios del servicio, la comprensión de la realidad actual, la importancia de la participación o la complejidad del propio proyecto educativo que están desarrollando.

La sociedad actual requiere que los universitarios sean personas participativas en la sociedad y comprometidas con ésta, que posean una amplitud de miras social y profesionalmente, que den significado a los conocimientos académicos, y que aprendan a vivir valores como la solidaridad, el respeto o el compromiso. Para facilitar estos aprendizajes se precisa disponer de oportunidades educativas $y$, entre ellas, consideramos que las experiencias educativas que se desarrollan a través del aprendizaje servicio cumplen con los requisitos para llevarlos a cabo.

Finalmente cabe apuntar que las grandes oportunidades que el ApS ofrece serán posibles si las políticas educativas e institucionales de nuestras universidades apuestan por ello. Es necesario reflexionar institucionalmente sobre este tipo de prácticas educativas si se apuesta que este tipo de aprendizajes sean objetivos sistematizados de nuestras universidades o por el contrario, sean aprendizajes dejados al azar que solo algunos llegarán a alcanzar. 


\section{Metodología}

La relevancia que puede llegar a tener el aprendizaje servicio en el contexto universitario, tal y como se describió en el apartado anterior, llevó a plantearnos cómo se estudia esta metodología a través del análisis de las tesis doctorales defendidas en las universidades españolas durante las dos últimas décadas, además de observar la tendencia en las investigaciones sobre ApS.

Los datos han sido recogidos a través de la consulta de la Base de datos de Tesis Doctorales (TESEO) del Ministerio de Educación, Cultura y Deporte. La búsqueda se ha realizado tomando como criterios el período seleccionado (cursos académicos desde el 1999-2000 hasta el 2015-2016) y los términos aprendizaje-servicio, aprendizaje servicio y service learning. Por su frecuencia de uso se ha incluido el término en inglés, además de los dos en castellano (con y sin guión).

No se ha utilizado el tesauro de TESEO porque no hay una coincidencia plena en las materias a las que está vinculada la temática del aprendizaje servicio. Las opciones son múltiples, entre las que destacan, por orden decreciente en función de los descriptores empleados (ver tabla 2): métodos pedagógicos, teoría y métodos educativos, teorías educativas, sector de la educación, educación superior, asesoramiento y orientación educativa, pedagogía, educación básica. Pero, también se pueden encontrar materias tan diversas como planes de estudios (Código UNESCO: 580103), relaciones urbano- rurales (Código UNESCO: 322909) o política cultural (Código UNESCO: 590202), entre otras, con una única mención.

\begin{tabular}{|l|l|l|}
\hline \multicolumn{1}{|c|}{ Descriptor } & \multicolumn{1}{|c|}{$\begin{array}{c}\text { Código } \\
\text { UNESCO }\end{array}$} & $\begin{array}{c}\text { No Tesis } \\
\text { Doctorales }\end{array}$ \\
\hline $\begin{array}{l}\text { Métodos } \\
\text { pedagógicos }\end{array}$ & 580107 & 6 \\
\hline $\begin{array}{l}\text { Teoría y métodos } \\
\text { educativos/Métodos } \\
\text { educativos }\end{array}$ & $580100 / 610402$ & 5 \\
\hline $\begin{array}{l}\text { Teorías educativas } \\
\text { Sector de la } \\
\text { educación } \\
\text { Educación superior }\end{array}$ & 580104 & 3 \\
\hline $\begin{array}{l}\text { Asesoramiento y } \\
\text { orientación } \\
\text { educativa }\end{array}$ & 580204 & \\
$\begin{array}{l}\text { Pedagogía } \\
\text { Educación básica }\end{array}$ & 580303 & 2 \\
\hline
\end{tabular}

Tabla 2: Descriptores más frecuentes a los que se vinculan las Tesis Doctorales sobre ApS. Fuente: elaboración propia

Los datos se han identificado efectuando un análisis de contenido a través de la revisión de los títulos, los descriptores y los resúmenes, valorando la productividad de la temática, las principales características generales y metodológicas y los temas estudiados.

Las variables analizadas han sido título de la tesis, año de lectura, género de la autoría, universidad donde se defendió la tesis, temática y metodología de investigación.

\section{Resultados}

De la búsqueda de tesis doctorales llevada a cabo mediante las palabras clave aprendizaje-servicio, aprendizaje servicio o service learning en la base TESEO se han obtenido 16 trabajos durante los últimos 16 años (curso 
académico 1999-2000 a 2015-2016).

En los primeros nueve años revisados solo figura una tesis sobre aprendizaje servicio (en 2005) y hasta el año 2010 no se observa una pequeña evolución de carácter ascendente en la productividad de tesis (ver figura 1 ), correspondiendo al año 2016 el mayor número de tesis defendidas (4), seguidos de los años 2013 y 2014 con 3 tesis presentadas cada uno.

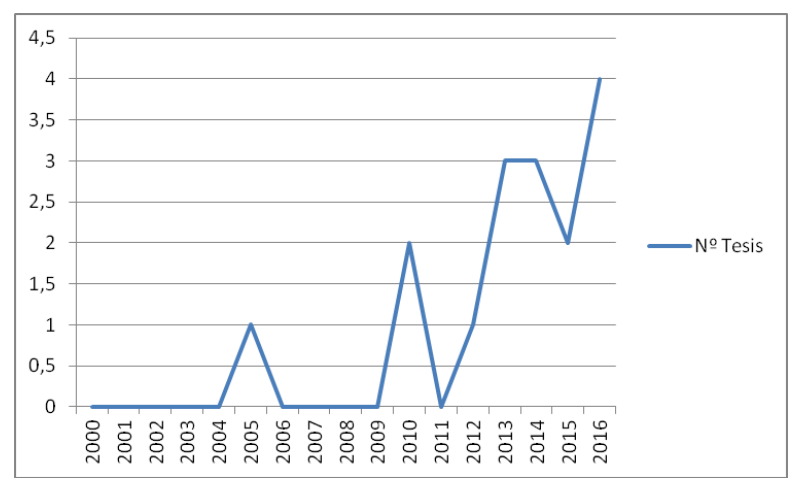

Figura 1: Evolución de la producción científica de Tesis Doctorales sobre Aprendizaje Servicio. Fuente: elaboración propia

Respecto al género en la autoría de las tesis, hay un predominio claro de las mujeres $(62,5 \%)$ frente a los hombres $(37,5 \%)$.

Las universidades en las que se presentaron mayor número de tesis relacionadas con el ApS fueron la de Barcelona, la de Santiago de Compostela y la de Jaume I de Castellón (con 2 investigaciones) y con un trabajo figuran las Universidades de Almería, Deusto, Girona, Granada, Navarra, Valencia, Valladolid, la Universidad Autónoma de Madrid, la Complutense de Madrid y la Universidad Nacional de Educación a Distancia.
En cuanto al análisis de la metodología de investigación empleada en las tesis analizadas, hay que indicar, en primer lugar, que el $37,5 \%$ de las mismas no incluyeron referencias sobre este aspecto en la información que figura en la base TESEO. En las tesis restantes los diseños más utilizados han sido la investigación evaluativa con un $18,75 \%$, seguidos por el mixto, el cuasi-experimental y el estudio de casos (los tres enfoques con un $12,5 \%$ ) $y$, en último lugar, el no experimental $(6,25 \%)$. Para la recogida de información se aplicaron, principalmente, el análisis documental, los cuestionarios, las entrevistas y los grupos de discusión.

Cabe destacar que las investigaciones sobre el aprendizaje servicio llevadas a cabo en las tesis doctorales se enfocaron prioritariamente en los estudiantes, con especial incidencia en los universitarios, seguidos de los que cursan enseñanza secundaria y, por último, los que se encuentran en la etapa de educación primaria. Aparecen también otros destinatarios (voluntariado, profesionales de recursos públicos y representantes de entidad social y administradores y operadores culturales). En la tabla 3 se muestra esta distribución, teniendo en cuenta que una misma tesis puede integrar más de un colectivo de los destinatarios comentados. 


\begin{tabular}{|l|c|}
\hline Destinatarios & $\begin{array}{c}\text { No Tesis } \\
\text { Doctorales }\end{array}$ \\
\hline Alumnado universitario enseñanza & 11 \\
\hline $\begin{array}{l}\text { Alumnado de primaria } \\
\text { secundaria }\end{array}$ & 2 \\
\hline Alumnado de enseñanza pris & 3 \\
\hline Otros
\end{tabular}

Tabla 3. Destinatarios de las propuestas de ApS en las Tesis Doctorales analizadas. Fuente: elaboración propia

Las temáticas en las que se ha centrado el interés de la comunidad científica sobre la metodología del aprendizaje servicio en el período revisado están muy relacionadas con dos factores. El primero se refiere a los diversos beneficios que aporta este enfoque en dimensiones como la sociedad del conocimiento, la formación cívica y la participación ciudadana, la responsabilidad social, la ciudadanía activa, el emprendimiento social, la dimensión social de la Universidad, los aprendizajes académicos, la orientación educativa, las relaciones colaborativas entre la escuela y la comunidad o la salud de los estudiantes. Y, el segundo se vincula con propuestas de implementación y evaluación de programas de aprendizaje servicio en diferentes contextos, especialmente educativos.

\section{Conclusiones}

En los últimos años se ha incrementado una mayor concienciación sobre la responsabilidad de las universidades e instituciones de educación superior a la hora de aportar su caudal de conocimientos y recursos al servicio de la comunidad.

La educación superior debe reforzar sus funciones de servicio a la sociedad, y más concretamente sus actividades encaminadas a erradicar la pobreza, la intolerancia, la violencia, el analfabetismo, el hambre, el deterioro del medio ambiente y las enfermedades, principalmente mediante un planteamiento interdisciplinario $y$ transdisciplinario para analizar los problemas y las cuestiones planteados (UNESCO, 1998).

De este modo el compromiso social que presenta la educación superior ha llevado a repensar los planteamientos metodológicos y a mostrar las fortalezas de la propuesta pedagógica del aprendizaje servicio como un enfoque de articulación y encuentro de intencionalidades y culturas institucionales.

Tal y como hemos venido presentando en este trabajo de investigación, el aprendizaje servicio resulta ser pues una metodología particularmente innovadora ya que contribuye a superar la brecha entre teoría y práctica y articula las misiones de extensión, investigación y docencia aportando beneficios simultáneamente a la responsabilidad social y la excelencia académica.

Al conectar las prácticas solidarias con posibilidades de acción profesional, los proyectos de aprendizaje servicio pueden contribuir a formar graduados universitarios conocedores y comprometidos con las necesidades sociales.

Con todo ello podemos concluir que el estudio realizado nos ha mostrado el 
interés creciente en el ámbito académico y científico por el aprendizaje servicio ya que se trata de una metodología pedagógica que promueve el desarrollo de numerosas y muy diversas competencias a través de actividades educativas que incluyen el servicio a la comunidad.

El ApS se caracteriza por ser una propuesta innovadora, que puede transformar el proceso de adquisición de conocimientos y convertirse en un camino participativo de educación cívica y de formación en valores para la ciudadanía.

El contexto universitario destaca en las tesis analizadas como uno de los prioritarios para el desarrollo de programas de ApS.

Se puede destacar la idoneidad de esta metodología para aprender contenidos curriculares, para cambiar la percepción del alumnado sobre la concepción de la universidad, para desarrollar valores y actitudes relacionadas con la ciudadanía, para gestionar cambios en la formación inicial de los estudiantes y de los futuros graduados (reforzando las dimensiones de competencia profesional, por ejemplo), entre otras finalidades.

$Y$, aunque se percibe una tendencia creciente en el campo científico y académico por el ApS, constatable en la progresión en el número de tesis doctorales defendidas en España en los últimos años con esta temática como foco de investigación, y se tienen los "estímulos que pueden ser aprovechados para el desarrollo de proyectos de aprendizaje-servicio (...) no puede decirse que esta metodología de trabajo educativo haya calado en el sistema, hablemos del nivel de enseñanza que prefiramos. Tampoco desde luego en la Universidad" (Santos, Sotelino y Lorenzo, 2015, 93-94).

Por lo que resulta necesario, como propuesta de futuro, incorporar este enfoque metodológico en todas las etapas educativas. Para ello, será de gran ayuda la labor de difusión de experiencias y buenas prácticas de aprendizaje servicio que puedan llevar a cabo los investigadores y estudiosos de esta temática.

\section{Referencias bibliográficas}

Agencia Nacional de Evaluación de la Calidad y Acreditación (ANECA) (2005). Libro Blanco para el título de Grado en Magisterio. Madrid: ANECA.

Andrea, F., y Lidón, M. (2010). El Aprendizaje Servicio en la Universidad: Una estrategia en la formación de ciudadanía crítica. REIFOP, 13(4). Recuperado de: http://www.aufop.com.

Bolívar, A. (2008). El discurso de las competencias en España: educación básica y educación superior. Revista de Docencia Universitaria, 6(2), 1-23.

Delors, J. (1996). Informe Delors. La educación encierra un tesoro. Madrid: UNESCO-Santillana.

Hersh, L., Simone, D., Moser, U., y Konstant, J. (1999). Proyectos sobre competencias en la OCDE. París: OCDE.

Jacoby, B. et al. (1996). ServiceLearning in Higher Education: Concepts and Practices. San Francisco, CA: Jossey-Bass. 
Ley 45/2015, de 14 de octubre, de Voluntariado, BOE núm. 247, de 15 de octubre de 2015.

Piqué, B., y Forés, A. (2012).

Propuestas metodológicas para la educación superior. Barcelona:

Universitat de Barcelona. Recuperado de http://hdl.handle.net/2445/30702.

Puig, J. M., Batlle, R., Bosch, C. et al. (2006). Aprenentatge Servei.

Barcelona: Octaedro y Fundació Jaume Bofill.

Puig, J. M., Batlle, R., Bosch, C., y Palos, J. (2007). Aprendizaje y servicio para la adquisición de las competencias básicas. Barcelona: Octaedro.

Real Decreto 1791/2010, de 30 de diciembre, por el que se aprueba el Estatuto del Estudiante Universitario. BOE núm. 318, de 31 de diciembre de 2010.

Santos, M. A., Sotelino, A., y Lorenzo, M. (2015). Aprendizaje-servicio y misión cívica de la universidad.

Barcelona: Octaedro.

UNESCO (octubre, 1998). Declaración Mundial sobre la Educación Superior en el siglo XXI. Visión y acción. Trabajo presentado en la Conferencia Mundial sobre la Educación Superior, París.

Recuperado de http://unesdoc.unesco.org/images/001 1/001163/116345s.pdf. 